\title{
Un sistema mitico in continua evoluzione: le grandi parodie Disney
}

\author{
Andrea Cannas \\ Università degli Studi di Cagliari \\ deandrade@libero.it \\ "il fumetto è narrazione espressa attraverso arte sequenziale, un \\ medium che risale a 17 mila anni fa, \\ alle pitture rupestri di Lascaux" \\ (Sheldon Cooper in The Big Bang Theory)
}

\begin{abstract}
Il fitto dialogo intertestuale con i classici di ogni tempo è stato inteso dagli autori della Disney Italia come una pratica di cannibalizzazione delle fonti; d'altra parte il confronto con la dimensione letteraria, estendendosi nel tempo - dalla fine degli anni Quaranta a oggi -, ha lasciato un'impronta indelebile sulle Grandi Parodie: per un verso esse hanno acquisito uno spessore testuale inedito, riconnettendosi alle grandi matrici narrative in cui affonda le proprie radici il più vasto mondo della finzione; per l'altro hanno costituito un formidabile banco di prova per la variegata compagine Disney: gli eroi del fumetto, da Topolino a Paperino, si sono cimentati nell'impresa di interpretare il ruolo e assumere le fattezze dei più rinomati protagonisti della pagina letteraria mostrando al contempo una grande duttilità e una spiccata personalità. Il complesso dei personaggi Disney funziona in definitiva come un sistema mitico: il corpo delle loro storie costituisce un intero aperto a un continuo accrescimento ed è in effetti costantemente aggiornato da una pluralità di autori che rende il sistema perennemente sincronico col tempo dei lettori.
\end{abstract}

Parole chiave: Fumetto. Disney. Grandi Parodie. Mito. Intertestualità.

RESUMO: O estreito diálogo intertextual com os clássicos de várias épocas foi entendido pelos autores da Disney Itália como uma prática de canibalização das fontes, que proporcionou o confronto com a dimensão literária, estendendo-se no tempo - do fim dos anos 40 a hoje -, e deixou uma marca indelével nas Grandes Paródias: por um lado, estas adquiriram um peso textual inédito, reconectando-se com as grandes matrizes narrativas nas quais fincam as suas raízes no mais vasto mundo da ficção; por outro ainda constituíram um formidável teste para a multifacetada equipe Disney: os heróis dos quadrinhos, de Mickey a Donald, cimentaram-se na empreitada de interpretar os papéis e assumir os perfis dos mais renomados protagonistas das páginas literárias, demostrando, ao mesmo tempo, uma grande flexibilidade e uma marcada personalidade. $\mathrm{O}$ conjunto dos 
personagens Disney funciona definitivamente como um sistema mítico: o corpo das suas histórias constitui um todo, aberto em uma contínua evolução, e é, de fato, constantemente atualizado por uma pluralidade de autores que torna tal sistema perenemente sincrônico com o tempo dos leitores.

Parole chiave: História em quadrinhos. Disney. Grandi Parodie. Mito. Intertextualidade.

ABSTRACT: An intense intertextual dialogue with the classics of all time has been intended by Disney Italian authors as a practice of source "cannibalization". Their long-lasting dialogue with the literary dimension left an indelible mark on the Grandi Parodie: on the one hand they acquired an unprecedented textual depth, connecting them to the major narrative roots of the fictional world; on the other hand, they represented a crucial test for the varied team of Disney characters: the heroes of comics, from Mickey Mouse to Donald Duck, undertook the remarkable feat of interpreting the roles of renowned literary characters, showing a great adaptability as well as a strong personality. Disney characters function fundamentally as a mythical system: their stories constitutes an ever-changing whole, constantly updated to the time of their readers.

Keywords: Comics. Grandi Parodie. Disney. Parody. Myth. Intertextuality.

\section{Non esistono arti minori}

Credo che nessuna persona ragionevole metta ancora in dubbio la piena autonomia del fumetto e l'opportunità di considerarlo, a tutti gli effetti, un'arte. Per la precisione, secondo l'ormai celebre definizione di Francis Lacassin ${ }^{1}$, esso costituirebbe la nona arte - a patto sia chiaro che l'ordinale non sta a indicare una posizione di retroguardia, in una graduatoria ipotetica e anacronistica: viceversa è indice di freschezza e attualità poiché fa riferimento alla piena consonanza del medium col nostro tempo. Non esistono, difatti, forme o generi di maggiore o minor pregio, ma tecniche e linguaggi peculiari che marcano le differenze e sono oggetto di studi teorici e metodologici che fortunatamente vanno sempre più affinandosi. Per esempio, nel caso del fumetto occorre mettere in conto 
l'importanza predominante dell'elemento visuale e contestualmente il suo rapporto simbiotico con il testo scritto. Va contemplato, inoltre, il complesso di convenzioni le quali regolano e dispongono gli spazi - c’è difatti una strategia mediatica che si occupa di governare i riquadri, i bordi, le vignette e finanche i balloon: in fondo si tratta di segni iconografici che, non limitandosi semplicemente ad agevolare l'orientamento spaziale del lettore, veicolano la scansione/comprensione del tempo della storia ${ }^{2}$. Ciò premesso, non esiterei a valutare l'opportunità di considerare il fumetto come un'arte cugina in primo grado della letteratura: in effetti, se le vignette possono condividere con le pagine fitte di scrittura la presenza indispensabile di un fruitore che è un lettore ${ }^{3}$, dobbiamo prendere atto di una realtà consolidata che vede i libri a fumetti acquisire spazi significativi nelle librerie e addirittura ambire a concorrere ai premi letterari, con discrete possibilità di successo. La consanguineità favorisce una continua trasfusione di dati, di forme e di strategie compositive, ovvero si innesca inevitabilmente un dialogo che è al contempo intertestuale e intersemiotico.

\section{Il successo della scuola italiana Disney.}

Nel corso degli anni Settanta l'Italia scoprì d'essere il paese del mondo occidentale nel quale si leggevano più fumetti - in classifica generale alle spalle del solo Giappone - ma già nel decennio precedente una serie di indicatori aveva fornito segnali incoraggianti: crescevano le testate specializzate e si moltiplicavano i personaggi di successo; soprattutto si facevano sempre meno rari o fortuiti i casi di incroci e sovrapposizioni fra $i$ territori della letteratura e quelli delle tavole disegnate e parlanti: incontri variamente declinati che comunque davano conto della volontà di sperimentare nuove forme espressive su entrambi i versanti. Pochi anni dopo l'uscita di Apocalittici e integrati (1964), testo dedicato alle prospettive 
della cultura e letteratura di massa, firmato da un Umberto Eco poco più che trentenne, vedono la luce due opere che potremmo, in un certo senso, considerare speculari. Intanto la prima raccolta di Cosmicomiche, finalmente in volume nel 1965, a proposito delle quali Italo Calvino rivelava tra le eterogenee fonti d'ispirazione, accanto al Leopardi delle Operette morali e al teatro di Samuel Beckett, nientemeno che Popeye: le strisce per ragazzi suggeriscono all'autore l'invenzione della più seriale e fumettistica ${ }^{4}$ fra le sue creature, quell'ineffabile abitatore di sterminati cronotopi che è il signor Qfwfq. Quattro anni più tardi vede la luce Poema a fumetti, capolavoro che nasce dal connubio di scrittura testuale e visuale di Dino Buzzati, fra i primi rappresentanti in Europa (e nel mondo) di un nuovo genere, quello del graphic novel: in 208 tavole a colori l'autore rivisita la vicenda di Orfeo (il poeta per antonomasia) e della sfortunata Euridice (il cuore stesso della sua poesia), vale a dire che l'opera si confronta con uno dei miti dalla maggiore stratificazione letteraria, andando consapevolmente a infittirne l'intrico delle varianti.

In quegli stessi anni fiorisce in tutto il suo fulgore anche la stagione della Disney Italia, ovvero la scuola di autori che, vantando nomi come quelli di Guido Martina, Luciano Bottaro, Romano Scarpa e Giovan Battista Carpi, arriverà a produrre di lì a poco (senza peraltro poterli firmare individualmente) il 75\% dei fumetti Disney che il bel paese esporta in tutto il mondo: fiore all'occhiello della scuola, e ancora oggetto di culto per svariate generazioni di lettori, s’imponeva il raffinato ciclo delle Grandi Parodie, lunga teoria di riscritture che aveva vissuto l'abbrivo con L'inferno di Topolino $(1949)^{5}$ - in uno dei primi numeri della (all'epoca) giovanissima testata di Topolino. Fin dal principio, e in corrispondenza di alcune delle più significative tappe evolutive, il fumetto (e non solo quello Disney) ha tratto ispirazione e linfa vitale dai classici della storia letteraria ${ }^{6}$; per esempio lo 
stesso Zio Paperone attingeva nome (Uncle Scrooge) e carattere dall'avido Ebenezer Scrooge del Canto di Natale di Dickens. Il grande Carl Barks d'altro canto mostrò di non disprezzare affatto l'opportunità di riplasmare alcuni fortunati soggetti letterari, penso per esempio allo splendido Zio Paperone e il vello d'oro - The Golden Fleecing, del 19557. E tuttavia, la strategia pianificata dalla Disney Italia non si affida a episodi sporadici ma agisce, in breve volgere di tempo, in modo sistematico, come confermano i numeri in continua crescita almeno a partire dai primi anni Sessanta.

Il fitto dialogo intertestuale con i classici di ogni tempo e luogo è stato sovente inteso dagli sceneggiatori e dai disegnatori delle storie a fumetti come una pratica di cannibalizzazione delle fonti. D'altra parte, il confronto prolungato con la dimensione letteraria ha lasciato un'impronta indelebile sulle Grandi Parodie: esse hanno acquisito uno spessore testuale inedito, il quale dipende sostanzialmente dall'evidenza per cui quelle storie si sono riconnesse alle grandi matrici narrative in cui affonda le proprie radici il più vasto mondo della finzione. La qualità finale del prodotto, si pensi anche solo a titolo esemplare alle terzine vergate da Guido Martina per l'Inferno di Topolino, era tale da mettere d'accordo grandi e piccini: il gradimento condiviso derivava da una tipologia d'opera che era concepita e si prestava a più livelli di lettura. Il già citato Dino Buzzati ebbe modo di dire la sua discorrendo in generale dell'universo paperinesco - nella breve prefazione al volumetto Mondadori Vita e dollari di Paperon de' Paperoni (1968), nella quale rigettava elegantemente $i$ preconcetti che incombevano sulle storie a fumetti, relegate nell'ambito riduttivo dei prodotti per l'infanzia:

Colleghi e amici, quando per caso vengono a sapere che io leggo volentieri le storie di Paperino, ridono di me, quasi fossi rimbambito. Ridano pure. Personalmente sono convinto che si tratta di una delle più grandi invenzioni narrative dei tempi moderni. Lasciamo pur stare la vertiginosa fantasia e ingegnosità delle vicende, ammirevoli in un mondo dove la regola quasi sovrana dei romanzi è la noia. Sono i due protagonisti, Paperino e Paperon de' Paperoni, a fare la gloria maggiore di Walt Disney. La loro statura, umanamente parlando, non 
mi sembra inferiore a quella dei famosi personaggi di Molière, o di Goldoni, o di Balzac, o di Dickens.

\section{Il sistema mitico dei personaggi Disney}

Lo spessore dei due protagonisti, vere star di Paperopoli, non discendeva da un lavoro di scavo psicologico semplicemente perché un'attitudine di questo tipo non rientrava nelle finalità del lavoro dei loro creatori: piuttosto è il risultato dellininterrotto sedimentarsi di storie che da decenni ormai si riavvitano ingegnosamente su se stesse. Un po' come accade per i grandi eroi del mito, la varietà degli intrecci e delle situazioni - considerevole certo, ma non illimitata - insieme al costante ri-aggiornamento perpetuato da una pluralità di autori determinano sia la personalità composita sia l'umana contraddittorietà dei Paperi. Per citare il Lotman della Semiosfera (1985, pp.204-205), in un discorso inerente lo sviluppo della narrazione mitica, si potrebbe dire che il corpo delle loro storie costituisce un intero aperto all'accrescimento, come un cespo di cavolo in cui, susseguendosi una dopo l'altra, ciascuna foglia assomigli ma allo stesso tempo si distingua almeno un poco da quella precedente. Siccome quei personaggi vivono, come accade il più delle volte nei fumetti seriali, in una perenne sincronicità con i lettori all'anagrafe Topolino e Paperino potrebbero essere ormai i nonni di quelli più giovani ${ }^{8}$ - è necessario che anche il contesto delle loro vicende si adegui di volta in volta a una realtà in costante mutamento. Tutto ciò non sembra spaventare i creatori dell'universo Disney: d'altra parte, come avrebbe detto Blumenberg (1991, pp.219-220) a proposito del mito, non importa tanto quello che esisteva all'inizio quanto ciò che "resiste" alla fine del processo evolutivo, ciò che è in grado di soddisfare il gusto e l'orizzonte d'attesa del pubblico. Ad ogni modo, gli eroi del fumetto, Paperi e Topi, disegnano un percorso e vivono entro un tempo circolare - cioè ogni volta ricominciano da capo, poiché sono stati sottratti alla stagione del loro declino così come 
alla morte: alla stregua degli eroi o semidei del mito, sono condannati a tornare sui loro passi.

C'è ancora un aspetto che va tenuto in considerazione. Quando gli sceneggiatori e i disegnatori sono chiamati a re-interpretare un soggetto che abbia dato corso a una consistente parabola letteraria, essi propongono una nuova versione che non solo si confronta ma in un certo senso contesta il classico di turno: quando la sfida dell'intertestualità raggiunge un tale livello di competizione, gli autori tendono ad adottare gli stratagemmi tipici della scrittura apocrifa. Per esempio fingono di confutare il modello sublimando la loro variante al rango di archetipo del discorso mitico. È la ragione per cui Zio Paperone, nella parodia dedicata alla Locandiera, può affermare al cospetto dei nipoti “Ecco ragazzi, qui c'è la vera storia della Locandiera [...] Da qui Goldoni si è ispirato per scrivere la sua commedia [...] I protagonisti erano i nostri avi" (Zio Paperone e la locandiera, 1989). In effetti non va trascurata l'evidenza per la quale Paperi e Topi possono vantare un albero genealogico sconfinato: l'osservatore che ambisse a ripercorrerne le ramificazioni potrebbe contemplare i campioni delle due famiglie vivere perfettamente a loro agio, e con immutata coerenza, anche al tempo degli antichi egizi o sotto l'impero di Roma: il viaggio a ritroso nel tempo consente di ampliare a dismisura il bacino di racconti possibili, i quali oscillano - è ciò che accade anche nelle Grandi Parodie - fra l'esotismo centrifugo dell'avventura fuori-contesto e la forza centripeta garantita dalla riconoscibilità dei caratteri ${ }^{9}$. Tutto ciò era già stato intuito dal geniale inventore di Paperopoli, Carl Barks, il quale in effetti designava l'universo dei Paperi come una mappa spazio-temporale i cui confini continuano a cambiare e a espandersi ${ }^{10}$.

\section{Il vampirismo non è una carriera.}


Il principio della riscrittura è dunque il più prolifico motore narrativo atto a generare storie che sono al contempo, agli occhi del fruitore, in parte vecchie e in parte nuove, com'è appunto il risultato delle variazioni all'ordito dei miti cui si accennava. E non sarebbe affatto aleatorio sostenere che le espressioni popolari dell'arte sono state spesso incentivate dai processi della riscrittura così come, in maniera speculare, non è difficile verificare fino a che punto la poesia "colta" ri-attivi e dia sostanza letteraria a materiali che avevano già vissuto un'ampia circolazione orale. Se ogni autore può essere considerato alla stregua di un vampiro ${ }^{11}$ che succhia il sangue perennemente in circolo nella sterminata biblioteca universale, è pur vero che la creatura oggetto di vampirismo, una volta consumata la liturgia, non sarà più uguale a se stessa. Le Grandi Parodie della Disney Italia hanno sistematicamente pianificato quanto era già stato intuito dai maestri americani (Cfr. FAETI 1986, pp.93-94), i quali, come si diceva poc'anzi, non avevano disdegnato alcune fugaci ma paradigmatiche escursioni nel campo della finzione letteraria, con il preciso intento di riattualizzare formule di già consolidato successo. In gioco c'è il bonus della riconoscibilità: l'eco o il riverbero del capolavoro sollecita la curiosità di un pubblico che travalica i confini di genere, ma è palpabile anche l'ambizione degli autori a confrontarsi con l'aristocrazia della critica. Fatto sta che le arti novelle, o presunte tali, spesso impegnate all'esordio in una sorta di rito iniziatico, hanno in passato ricercato la benedizione di un padrino illustre, fino a chiamare in causa, in uno spazio condiviso per la loro consacrazione, i successi portati in auge da quelle forme espressive che già vantavano una tradizione consolidata. Si pensi a L'Inferno, film muto del 1911 diretto da Francesco Bertolini, Giuseppe de Liguoro e Adolfo Padovan, primo lungometraggio nella storia del cinema italiano, il quale adattò per il grande schermo la prima cantica della Commedia di Dante - senza disdegnare, in aggiunta, l'omaggio 
figurativo alle illustrazioni di Gustave Doré.

Conquistate le luci della ribalta, l'approccio non potrà che essere più maturo e disinvolto: si può anzi affermare, a mo’ di postulato, che la gravità del tradimento perpetrato nei confronti del modello originale sarà direttamente proporzionale al grado di autonomia e consapevolezza conquistata sul campo dal nuovo medium.

Per altro verso, è già stato notato come il fumetto goda di una libertà d'azione che si manifesta concretamente nel poter mescolare con una certa disinvoltura alto e basso, surrealismo e canzonette, figure archetipe e grafica pubblicitaria: il suo statuto dinamico ha modulato un mezzo terribilmente congeniale al nuovo millennio per la velocità di ricezione, l'impatto del linguaggio visivo e la possibilità di fruizione distratta (cfr. LA PORTA, 1999) - il quale è tuttavia capace di dire cose che non sempre si dispongono in pacata sintonia con il contesto cui pertiene il lettore: sotto questo aspetto, il fumetto è non solo un valido strumento per indagare le forme e la costruzione dell'immaginario nella cultura popolare, ma anche una delle modalità espressive più spregiudicate nel momento in cui problematizza i modelli sociali e culturali vigenti, fino al punto di denunciarne le storture. Con l'effetto benefico, in definitiva, di rimetterli in discussione.

\section{L'eroe multiforme, a volte ingegnoso, delle parodie Disney: Paolino Paperino}

Basta una semplice analisi statistica degli adattamenti che vedono protagonisti i Paperi al confronto con i Topi ${ }^{12}$ per comprendere come il vero protagonista delle parodie Disney sia Paolino Paperino. È il precipitato della sua propensione a imporsi quale cittadino/papero medio per eccellenza, nel quale si può rispecchiare (se non proprio identificare) la maggior parte dei lettori - purché sia chiaro che quella mediocrità deriva 
dalla compatibilità degli estremi, ovvero dalla compresenza del tragico e del comico $^{13}$, del sublime e del triviale mai davvero riconciliati, dote che gli garantisce un'eccezionale versatilità ${ }^{14}$. Il pigro e nullatenente nipote del miliardario Paperone è l'anti-eroe per eccellenza, e la sua inseparabile casacca da marinaio gli calza a pennello come il destino bizzarro e a volte cattivo che lo perseguita: nel complesso delle parodie egli costituisce al contempo la sublimazione e la confutazione dell'Ulisse ${ }^{15}$ dal multiforme ingegno. Veste effettivamente i panni dell'eroe omerico nella Paperodissea (1961), dove l'opportunità d'aguzzare la mente gli consente, per una volta, di gabbare il fortunato cugino Gastone - il quale insidiava una Paperina/Penelope indaffarata nella tessitura di mostruosi maglioni di lana; nella Paperiade (1959), invece, Paperino interpreta almeno tre ruoli: è Menelao defraudato, Achille in preda all'ira funesta, ma è soprattutto l'artefice del Cavallo in quella che risulta essere una performance dal sapore squisitamente carnevalesco. Ne L'Inferno di Topolino, dopo varie incursioni, si prendeva il proscenio in qualità di abitante della fiamma biforcuta, dove palesava la sua natura un poco schizofrenica e (in conformità con l'originale dantesco) sostanzialmente ambigua.

Paperino è insomma il pulsante d'innesco del meccanismo parodico, la cui finalità ultima - come sosteneva il Michail Bachtin de L'opera di Rabelais e la cultura popolare - è pervenire allo scoronamento dell'eroe: per ottenere il risultato ottimale presuppone e prende le mosse dalla perfetta conoscenza dell'opera da sovvertire, in un processo che contempla un discreto grado di interazione e complicità fra autore e lettore. Le Grandi Parodie a fumetti da un lato traggono costante alimento da una vena letteraria che, esibita in superficie e allusa fin dai titoli, continua ad agire in profondità; dall'altro si giovano della innata tendenza al travestimento burlesco da parte di quel Donald Duck che nasceva come spalla del topo più celebrato d'America - e 
tuttavia mostrava fin dalla più tenera età d'essere naturalmente portato all'insubordinazione. Erede della commedia dell'arte, campione della fuga come Pinocchio, l'eclettico Paolino Paperino è tuttavia capace di interpretare dentro lo stesso dramma, ricucendoli assieme, il ruolo del servo sciocco e di quello astuto.

\section{Tre modalità di approssimazione ai testi classici}

Il testo classico rivisitato nelle tavole a fumetti viene assimilato, di volta in volta, attraverso differenti modalità di approssimazione temporale. La prima è quella che ho altrove (***, pp.30-47) definito parodia con cornice, caratterizzata da un prologo dove i Paperi (o i Topi) vivono, come loro abitudine, in uno spazio-tempo che è perlopiù coincidente o comunque identificabile con la dimensione in cui vive il lettore. Il ricorso a espedienti rituali $\mathrm{i}$ quali hanno a che fare con $\mathrm{i}$ miti fondativi del racconto - per esempio il ritrovamento di un manoscritto, nel caso già citato della Locandiera: la famiglia si raccoglie intorno alla lettura ispirata del patriarca, o dello sciamano di turno - consente alla narrazione in primo piano di aprirsi a un ulteriore e più profondo livello finzionale, il quale consiste di fatto nel riadattamento vero e proprio, con gli eroi disneyani chiamati a interpretare $i$ diversi personaggi di spicco del testo letterario. La parodia vera e propria è dunque preceduta e introdotta sulla scena da una sorta di cornice declinata al presente: la netta cesura, mentre immerge il lettore in una stagione remota, dà adito a quello spiccato gusto tipicamente disneyano per l'esotismo, modulato questa volta sul piano delle coordinate temporali. In generale, quando attraverso l'artificio della cornice il racconto di primo grado genera un ulteriore spazio ancor più marcatamente fantastico (il racconto dentro il racconto), l'effetto può determinare nel lettore, o nello spettatore, l'opportunità di percepire quella soglia come una dimensione a sé 
prossima. Niente di nuovo sotto il sole: l'umana consistenza di Francesca e Paolo è palpabile proprio nel frangente in cui li sorprendiamo a sfogliare trepidanti le pagine del romanzo di Lancillotto e Ginevra, quando sono pronti a capitolare per consegnarsi a un grado totale, e almeno in quel caso fatale, di immedesimazione. Il meccanismo illusionistico e il suo gioco di scatole cinesi suggestionano il fruitore il quale a sua volta cede a una percezione realistica di quella zona di confine che precede il libero artificio dell'invenzione letteraria o fumettistica. E tuttavia, la cornice rappresenta anche il luogo dove può manifestarsi l'attrito, laddove la popolarità del fumetto sia chiamata a confrontarsi con il prestigio del modello letterario. Poiché il fumetto ha un proprio linguaggio a predominanza visiva, il codice convenzionale che regola e dispone gli spazi del fumetto avrà pure il compito di annunciare in modo appropriato la soglia che apre la frontiera fra il racconto-cornice e la narrazione di secondo grado, riorganizzando strategicamente i riquadri delle vignette. L'attraversamento della regione liminare è affrontato dunque con strumenti e artifici ormai convenzionali, eppure le conseguenze di quel movimento avranno non di rado significative ripercussioni tanto sul piano della trasmissione del modello di partenza quanto sul complesso dei personaggi Disney che lo reinterpretano.

La seconda tipologia, esemplarmente incarnata dall'indimenticato $I$ promessi paperi (1976), possiede i tratti peculiari della parodia in costume: $\mathrm{i}$ Paperi (o i Topi), senza alcun tipo di preambolo, vestono i panni e interpretano i ruoli dei protagonisti della fonte classica, sempre perfettamente individuabile a partire dal titolo dell'episodio. Si tratta probabilmente dell'immersione più indistinta entro una lontananza certo remota ma nutrita dalla memoria letteraria e visivamente evocata dai costumi indossati dai personaggi Disney. Il meccanismo parodico tende a mutuare le coordinate temporali dall'opera originale ma senza farsi 
condizionare più di tanto: non rinuncia a sfruttare le risorse di un immaginario che, per risultare veramente straniante, deve in prima istanza manifestarsi in forme cronotopiche vaghe e indistinte - l'eterno Medioevo della Disney, il cui marchio di fabbrica è l'onnipresente castello merlato.

L’ultima modalità d'approccio può essere intesa alla stregua di una parodia in tempo reale: i Paperi (o i Topi) vivono il loro ordinario presente nel quale potrà una volta di più identificarsi anche il lettore - senza concedere nulla agli assetti spazio-temporali della fonte letteraria, la quale viene dunque forzosamente traslata sull'orizzonte della contemporaneità. Se, in generale nelle Grandi Parodie, si crea sempre un rapporto dinamico fra l'apparato dei personaggi Disney e il complesso dei protagonisti dell'opera che viene rivisitata - una relazione che può sempre manifestare tensioni o attriti, e il rischio almeno potenziale del rigetto -, in questa specifica modalità il mondo a fumetti possiede di norma la capacità di piegare totalmente alle proprie esigenze, e alla propria tradizionale rete di rapporti, la fisionomia originaria dell'ipotesto.

Nei primi due casi - la parodia con cornice introduttiva e quella in costume, che peraltro sono le più frequenti - l'adattamento era già impostato secondo criteri ispirati alla libera reinterpretazione: nell'ultimo il riferimento al testo classico può essere ridotto ai minimi termini fino a risultare del tutto pretestuoso (cfr. la Paperopoli liberata del 1967). Ovviamente è possibile cogliere l'eco fievole che veicola ancora la voce del modello, e tuttavia è quasi esclusivo appannaggio del lettore onnivoro che possa vantare un discreto numero di frequentazioni letterarie.

La raffinatezza delle modalità strategiche che definiscono i vari approcci, coniugata alla loro messa a regime, ha prodotto una vastissima e variegata biblioteca alternativa, la quale viene da oltre settant'anni rinfoltita e aggiornata: gli adattamenti si moltiplicano e coinvolgono nuovi media come 
il cinema o il piccolo schermo; al contempo le vecchi parodie vengono periodicamente ripubblicate in nuove edizioni, anche di gran pregio, che si rivolgono ai lettori di tutte le età: i pezzi forti, come L'Inferno di Topolino, sono paradossalmente diventati a loro volta dei classici in virtù delle loro indiscutibili qualità testuali e visive. Ciascun volume è ormai intimamente connesso con altre opere, altri codici, altri linguaggi, in una trama di relazioni che è sorretta dal gusto del comico nel senso più maturo e consapevole: il comico cioè si fa sistema fino ad ambire all'autoparodia dell'universo Disney per un verso, per l'altro a coinvolgere il mondo cui pertiene il lettore.

Se poi tutto questo sia trionfo postumo dell'inarrivabile archetipo o piuttosto l'ineffabile liturgia che celebra la proliferazione irrefrenabile degli apocrifi, importa fino a un certo punto. Piuttosto, sembra che i nostri autori, gli uni e gli altri, tendano al puro movimento da cui derivano tutti i libri.

\footnotetext{
${ }^{1}$ Com'è d'obbligo, rimandiamo direttamente alla lettura del lavoro di Lacassin (1982).

2 Inevitabili i riferimenti a McCloud (1993); Eisner (1997); Groensteen (1999); Spiegelman (1999).

${ }^{3}$ E questo a prescindere dalla prevalenza del codice testuale o di quello visuale: ma si veda in proposito la posizione del già citato Groensteen (1999).

${ }^{4} \mathrm{Il}$ protagonista Qfwfq può in effetti vantare - con riferimento di nuovo all'Eco di Apocalittici e integrati - l'autorevole riconoscibilità dell'archetipo combinata a quella sostanza magmatica che si propaga in un numero sempre crescente e potenzialmente illimitato di varianti, qualità tipiche dell'eroe-mito delle serie a fumetti.

5 Nella relazione intertestuale con il prototipo dantesco, l'approccio di Guido Martina è ovviamente ludico; tuttavia non si faranno attendere altre clamorose operazioni che mettono in scena la Commedia a fumetti, le quali si muovono ben oltre l'orizzonte parodico: per citare un caso (recente) molto distante dall'universo della Disney Italia, basti pensare all'ottima reinterpretazione manga di Go Nagai, che copre tutte e tre le cantiche dantesche.

6 "La scelta di adoperare i capolavori immortali della letteratura mondiale di tutti i tempi come palinsesto di nuove suggestioni per le fantasie topo-paperinesche disneyane è un segno evidente della spiccata propensione letteraria della Disney Italia" (ARGIOLAS 2013, p.53).

${ }^{7}$ Sempre restando in ambito Disney, ma sul versante dei Topi, occorre indicare come punto di partenza delle parodie a fumetti il Mickey's Man Friday del 1935, rilettura ludica del capolavoro di Defoe.

${ }^{8}$ Mickey Mouse (1928) e Donald Duck (1934), difatti, non sono lontani dal compiere il loro primo secolo di vita.
} 
${ }^{9}$ Cfr. Storia e gloria della dinastia dei paperi pubblicato su Topolino dal n. 749 al n. 756 del 1970.

${ }^{10}$ Cfr. l'intervista al grande maestro americano reperibile in Andrae (2006, p.104).

${ }^{11}$ In uno degli ormai celebri prologhi della serie di cartoni animati The Simpsons, che avviava una puntata dedicata alla parodia dei classici della letteratura (era una puntata della tredicesima stagione che, tra gli altri episodi, metteva in scena una tanto gustosa quanto sintetica Odissea, nella quale inevitabilmente Homer vestiva i panni di Ulisse e Burt quelli di Telemaco), il piccolo Burt vergava sulla lavagna un monito valido per i rapsodi e i parodisti d'ogni tempo: "il vampirismo non è una carriera".

12 Almeno fino agli anni Settanta, le percentuali a favore dei Paperi sono imbarazzanti: oltre il $90 \%$ !

${ }^{13}$ È dunque il più degno interprete di un genere che Tosti (2011, p.45) definisce come il punto di congiunzione fra cultura alta e cultura bassa.

${ }^{14}$ Nonostante il topo sia l'animale anti-totemico per eccellenza, e quindi la star indiscussa della parodia zoomorfa, in ambito Disney le parodie interpretate dai Topi sono meno riuscite proprio a causa di una certa rigidità monocorde del protagonista. Al contrario, Paperino non solo è supportato da un cospicuo e variegato gruppo di comprimari - l'estesa famiglia dei Paperi - ma è egli stesso, in quanto sublimazione dell'uomo mediocre, capace d'interpretare un numero potenzialmente illimitato di ruoli. Se Paperopoli, con la sua riconoscibilissima sequela di emblemi della modernità, è lo spazio eternamente uguale a se stesso dove l'uomo mediocre viene quotidianamente perseguitato, l'antieroe tenderà a sviluppare una propensione centrifuga: il sistema delle Grandi Parodie gli fornirà nuovi spazi (e tempi alternativi) in cui manifestarsi.

${ }^{15}$ Cfr. Giorello e Cozzaglio (2013, p.17) per i quali è Topolino a palesarsi come "una sorta di Ulisse del nostro tempo, solo in apparenza appagato dalla quiete domestica". 


\section{Bibliografia}

ANDRAE, Thomas. Carl Bark sand the Disney Comic Book: Unmasking the Myth of Modernity. Jackson: University Press of Mississipi, 2006.

ARGIOLAS, Pier Paolo. La parodia Disney, fisionomia letteraria di un genere composito. In ARGIOLAS, Pier Paolo, CANNAS, Andrea, DISTEFANO, Giovanni V. e GUGLIELMI, Marina (a cura di). Le Grandi Parodie Disney, ovvero i Classici fra le nuvole. Roma: Nicola Pesce Editore, 2013, 51-92.

BACHTIN, Michail M. L'opera di Rabelais e la cultura popolare. Riso, carnevale e festa nella tradizione medievale e rinascimentale. Torino: Einaudi, 1979.

BLUMENBERG, Hans. Elaborazione del mito. Bologna: Il Mulino, 1991.

BOSCHI, Luca, GORI, Leonardo e SANI, Andrea. I Disney italiani. Dal 1930 al 1990, la storia dei fumetti di Topolino e Paperino realizzati in Italia. Bologna: Granata Press, 1990.

CANNAS, Andrea. Dall'Odissea a Guerre stellari: l'eterno ritorno dei Paperi. In Le Grandi Parodie Disney, cit., 15-49.

EISNER, Will. Fumetto e arte sequenziale. Torino: Vittorio Pavese, 1997.

FAETI, Antonio. In trappola col Topo. Una lettura di Mickey Mouse. Torino: Einaudi, 1986.

GIORELLO, Giulio e COZZAGLIO, Ilaria. La filosofia di Topolino. Parma: Guanda, 2013.

GROENSTEEN, Thierry. Système de la bande dessinée. Paris: PUF, 1999.

LACASSIN, Francis. Pour un neuvième art: la bande dessinée. Paris: Slatkine, 1982.

LA PORTA, Filippo. La nuova narrativa italiana. Travestimenti e stili di fine secolo. Torino: Bollati Boringhieri, 1999.

LOTMAN, Jurij M. La semiosfera. L'assimetria e il dialogo nelle strutture pensanti. Venezia: Marsilio, 1985.

MCCLOUD, Scott. Understanding Comics. The Invisible Art. New York: Kitchen Sink Press, 1993. 
SPIEGELMAN, Art. Comix, Essays, Graphics and Scraps. New York: Raw Books and Graphics, 1999.

TOSTI, Andrea. Topolino e il fumetto Disney italiano. Storia, fasti, declino e nuove prospettive. Latina: Tunué / Lapilli, 2011. 